\title{
Pulse-Discharge Plasmas for Plasma-Accelerator Applications
}

\author{
C. E. Clayton ${ }^{\mathrm{a}}$ C. Joshi ${ }^{\mathrm{a}}$, and N. C. Lopes ${ }^{\mathrm{b}}$ \\ ${ }^{a}$ Department of Electrical Engineering, University of California at Los Angeles \\ 405 Hilgard Avenue, Los Angeles, California 90095 \\ ${ }^{b}$ Grupo de Lasers e Plasmas, Instituto de Plasmas e Fusão \\ Nuclear, Instituto Superior Técnico \\ Av. Rovisco Pais, Lisboa 1049-001, Portugal
}

\begin{abstract}
For particle-beam-driven plasma wakefield accelerators, a long and fully-ionized plasma is desirable. We describe an experiment at UCLA to develop a prototype of such plasma using a pulsed-current discharge. Scaling of the plasma density with glass-tube diameter and with discharge-circuit parameters is currently underway. We have found that 4 Torr of Argon can be fully ionized to a density of about $1.3 \times 10^{17} \mathrm{~cm}^{-3}$ when the current density in the 1 inch diameter, 1.2 meter-long tube is around $2 \mathrm{kA} / \mathrm{cm}^{2}$, at least at one point along the discharge. The homogeneity of the plasma density in the longitudinal direction is crucial to prevent slippage of the driven plasma structures with the particles. Equally important are the transverse gradients since any dipole asymmetry in the transverse direction can lead to "steering" of the particle beam.[1] The longitudinal and transverse gradients may be a function of time into the discharge, the shape of the electrodes, the tube size, and the fractional ionization for a given fill pressure. These issues are currently under investigation.
\end{abstract}

Keywords: Ionization of Plasmas, Plasma Sources, Stark Broadening.

PACS: 52.25.Jm, 52.50.Dg, 52.70.Kz

\section{INTRODUCTION}

For particle-beam-driven plasma wakefield accelerators, a long and fully-ionized plasma is desirable. A meterscale plasma may be required for maximum energy gain of the accelerating particles. A uniform plasma is needed to maintain a constant length of the acceleration "bucket" to minimize longitudinal slippage of the accelerating charge. A fully ionized plasma can relax the requirements on the drive-beam's density and transverse emittance. For example, in the E-167 energy-doubling experiment,[2] performed at the Final Focus Test Beam (FFTB) facility at the SLAC National Accelerator Laboratory, the plasma was produced as the collective transverse electric field of the drive bunch field-ionized a long (up to $1.2 \mathrm{~m}$ ) column of Li vapor, producing electron densities approaching $3 \mathrm{x}$ $10^{17} \mathrm{~cm}^{-3}$. However, the maximum energy gain was experimentally found to occur with a plasma of length $85 \mathrm{~cm}$, limited by the emittance of the drive beam via "head erosion". This phenomena of head erosion-the loss of beam density at the front of the drive bunch - causes the location of the full-ionization point in the bunch to move backward as the bunch moves deeper into the Li column. Eventually, the particles that were accelerated and focusing over the first $85 \mathrm{~cm}$ found themselves in a location where the transverse focusing fields were no longer linear and they were deflected.[2] Because this process is related to field-ionization of the alkali metal vapor column, changing from $\mathrm{Li}$ to $\mathrm{Rb}$ (a lower ionization potential atom) would allow for longer acceleration lengths.

However, upcoming experiments at the recently-commissioned FACET facility,[3] also at SLAC, will put a new burden on the drive bunch for making long, tunnel-ionized plasmas. Here, rather than a highly longitudinally compressed bunch with its associated large transverse field, the beam in this upcoming experiment will be intentionally split into two bunches by (a) scattering away some of the central electrons via a blade located in a dispersive region of the FACET beam-line and (b) purposely not fully recompressing the resultant longitudinal current profile. Hence there will be a separate drive and witness bunch, but with the charge density in the drive bunch roughly four times lower than in the FFTB experiment. In addition, to place the witness bunch in the optimal phase of the wake created by the drive bunch, the density will be substantially lower than that used in FFTB; i.e., closer to $3 \times 10^{16} \mathrm{~cm}^{-3}$. At this density, the plasma length will need to be roughly $1.5 \mathrm{~m}$ to approach energy-doubling of the witness bunch. 
While not ruling out two-bunch experiments using tunneling ionization of, say, $\mathrm{Rb}$ in the near term, it is clearly prudent to build a long, uniform, preionized plasma to counter the now more-critical head-erosion issue. One track for doing this is to use a $10 \mathrm{TW}$, short-pulse laser coupled to an axicon lens to tunnel-ionize a meter-scale column of alkali metal vapor. This is discussed in Ref. [4] of these proceedings. Another track is to produce a plasma via a longitudinal discharge in a long, glass tube filled with a few T of Ar. Progress on producing a prototype of such a long discharge plasma source is the subject of this paper.

\section{DISHCHARGE TUBE}

Figure 1(a) shows a photograph looking along a 1.2m-long, borosilicate discharge tube; i.e., the plasma tube. For this image, the tube was 1.0 " outer diameter (OD) with about a $1.6 \mathrm{~mm}$ wall thickness. The yellow device surrounding the tube is a current monitor having a sufficiently high temporal resolution for these experiments. This current monitor is an important diagnostic. An optical fiber ( $10 \mathrm{~m}$ long with a $200 \mu \mathrm{m}$ glass core) is butted up against the tube and the local light thus collected is sent to a fast photodiode. This is another useful diagnostic.

The cathode electrode assembly is just visible at the end of the tube at the top of the image of Fig. 1(a). The twin cables connecting the pulsed, negative high voltage source (discussed in the next Section) come over from the left and is secured to a short length $(\sim 15 \mathrm{~cm})$ of $1 / 4$ " OD stainless steel (ss) tubing. One end of the ss tubing enters the discharge tube via an ultratorr fitting. Its cylindrical wall is locally chamfered down to a sharp edge for uniform electron emission. The glass discharge tube itself is sealed with a large-diameter ultratorr fitting. The other end of this ss tube is connected to an approximately $10 \mathrm{~m}$ length of blue polyethylene tubing, also visible at the top of Fig. 1(a), heading off to the right, and ending at a gas-mixing manifold. Shut-off valves and needle valves allow for adding a mixture of $\mathrm{Ar}$ and $\mathrm{H}_{2}$ into the discharge tube. The 10m length of plastic tubing helps to mix these gases and also prevents the discharge from traveling to the grounded metal gas-mixing manifold. A third diagnostic is a highvoltage probe that is in contact with the short ss tubing, outside of vacuum.
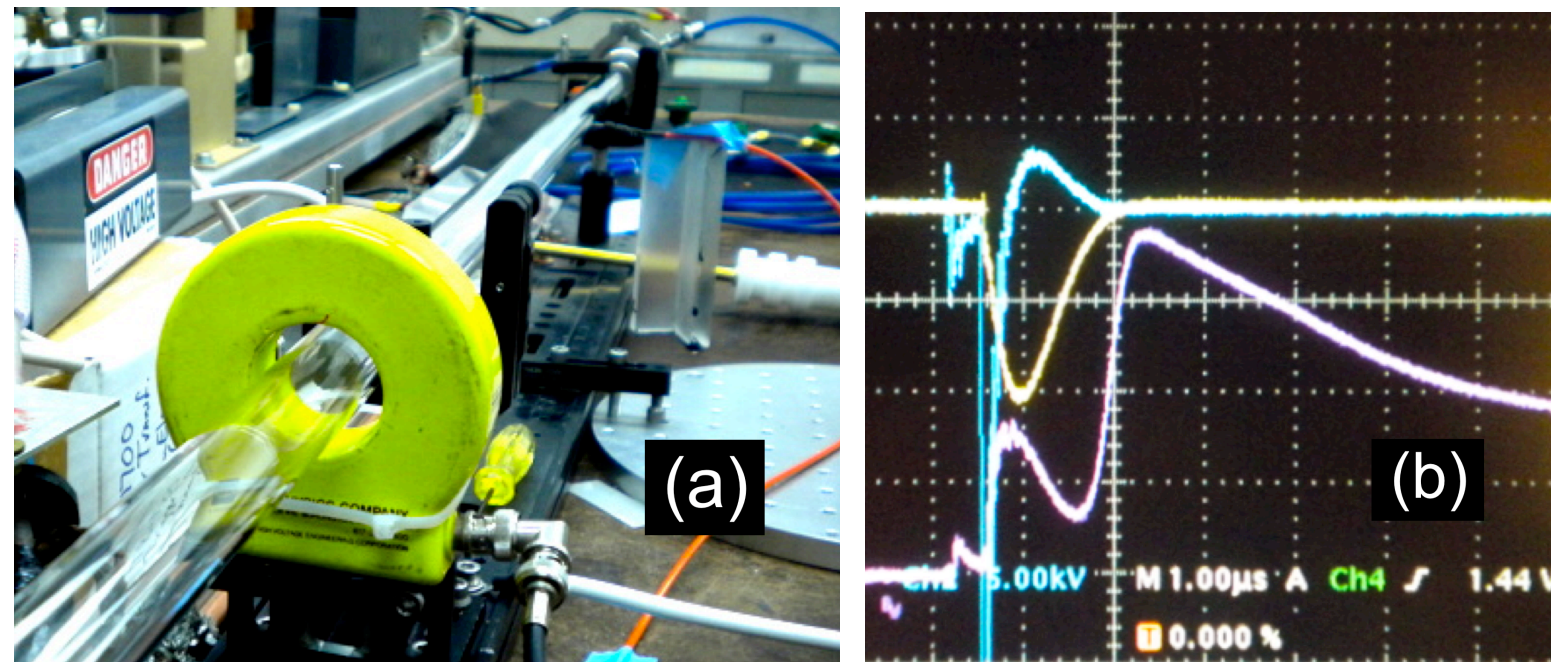

FIGURE 1. (a) A photograph looking along the 1.2m-long, 1" outer diameter discharge tube. The cathode-end is visible at the top of the picture and the yellow current monitor is in the foreground. (b) An image of an oscilloscope showing three traces. The cathode voltage is the uppermost trace; the one that shoots off-screen at the bottom of the image and has a scale of $5 \mathrm{kV} / \mathrm{div}$. The middle trace is the signal from the current monitor at about $0.4 \mathrm{kA} / \mathrm{div}$. The bottom trace is the broadband Ar emission (arbitrary units) picked up from the optical fiber. Note: the optical fiber signal is delayed by about 50ns with respect to the other traces due to the length of the fiber. For this shot, the tube diameter was 0.5 " $, \mathrm{C}=0.03 \mu \mathrm{F}, \mathrm{R}_{\mathrm{ext}}=16 \Omega$, and $\mathrm{V}_{0}=20 \mathrm{kV}$ with a resultant $\mathrm{J}_{\max }=$ $1.2 \mathrm{kA} / \mathrm{cm}^{2}$ (see text for these definitions).

At the other end of the 1.2m-long discharge tube is the anode assembly (not visible in Fig. 1(a)). It is essentially a 4-way cross (KF-25 connectors). One port holds the discharge tube via a large-diameter ultratorr fitting while the opposite port is a feed-through for a sharpened, 1/4" aluminum rod; the anode proper. One of the transverse ports is for a capacitance manometer to measure the nearly static neutral pressure within the discharge tube. The forth port has a KF-25 size, in-line valve that is subsequently connected to a 15 -liter vacuum chamber having a roughing pump 
and a turbo pump. The flow rate of Ar into the discharge tube is set to be greater than 10 times the largest-measured leak rate of the sealed-off glass tube and the inline valve is closed down so as to set the Ar pressure at the desired value-typically $4 \mathrm{~T}$. The $\mathrm{H}_{2}$ line has an ultra-fine needle valve and is set to add $20 \mathrm{mT}$ of $\mathrm{H}_{2}$ on top of the $4 \mathrm{~T}$ Ar. The purpose of the trace amount of $\mathrm{H}_{2}$ is for measuring the electron density via Stark broadening of neutral $\mathrm{H}$ line emission (see the Section on Density Measurements). The aluminum anode and the 4-way cross are connected to a 1 " wide mesh cable to carry the discharge return current back towards the cathode as will be discussed in the next section.

\section{DISCHARGE CIRCUIT}

It was not obvious at the beginning of this investigation as to the required voltage and current ratings of a discharge circuit capable of producing Ar plasma densities in the mid $10^{16} \mathrm{~cm}^{-3}$ range for tube diameters up to 1.5 ". Therefore, the most robust switching device on hand, a spark gap, was chosen as the switch. It is configured as a "trigatron" spark gap; that in which the ground electrode has a small hole in it into which the long electrode of a spark plug sits flush to, and centered in, this hole. It can be pressurized from 1 bar to $>5$ bar of (slowly flowing) synthetic air. The gap was adjusted so that the spark gap would operate at a charging voltage $\mathrm{V}_{0}$ as low as $4 \mathrm{kV}$ at a pressure of one bar to $30 \mathrm{kV}$ with about 5 bar of pressure. The DC charging supply, the trigger source, and other useful components were all conveniently borrowed from a Lumonics $103 \mathrm{CO}_{2}$ laser. A portion of this laser, with its protective cover removed, is seen in the upper left of Fig. 1(a). A variety of high-energy-density, low inductance Maxwell capacitors were on hand and used for energy storage.

The basic operation of the discharge is as follows. After setting the neutral $\mathrm{Ar}$ and $\mathrm{H}_{2}$ pressures, the spark gap is triggered at $1 \mathrm{~Hz}$. Then, after setting the spark gap pressure to a specified value, the high voltage is increased until a reliable and low-jitter $( \pm 100 \mathrm{~ns}$ maximum) operation of the circuit is achieved. The circuit inductance $\mathrm{L}$ and the internal resistance $\mathrm{R}_{\text {int }}$ were measured by firing the discharge in the $1.2 \mathrm{~m}$-long, $1 / 2$ " OD discharge tube with $4 \mathrm{~T}$ of $\mathrm{Ar}$ and at $20 \mathrm{kV}$ but without any external series resistance, $R_{\text {ext }}=0$. The resulting under-damped waveform decays exponentially with an e-folding rate $u=R_{\text {int }} /(2 L),[5]$ where $R_{\text {int }}$ is given by $R_{\text {int }}=R_{\text {plasma }}+R_{\text {inh }}, R_{\text {plasma }}$ is the resistance due to the plasma, and $\mathrm{R}_{\mathrm{inh}}$ is the sum of all other sources of series resistance; the inherent resistance. The damping factor $d$ is given by $d=R^{2} C /(4 L)$. Note: $d=1$ represents critically damping. The modified oscillation frequency $\omega$ is given by $\omega=[(1-\mathrm{d}) /(\mathrm{LC})]^{1 / 2}$. The measurements of $\mathrm{u}$ and $\omega$ gave $\mathrm{L}=2.8 \mu \mathrm{H}$ and $\mathrm{R}_{\text {int }}=3.2 \Omega$ for a capacitance $\mathrm{C}=0.03 \mu \mathrm{F}$. The calculated quantities are: $\mathrm{d}=0.03$, maximum current $\mathrm{I}_{\max }=1.6 \mathrm{kA}$, maximum current density within the tube $J_{\max }=2.2 \mathrm{kA} / \mathrm{cm}^{2}$, and the width of the first current pulse $\tau_{1 \mathrm{st}}=0.6 \mu$ s FWH where $\tau_{1 \mathrm{st}}$ is found from a Matlab script used to model the circuit. It was found that these values for $I_{\max }$ and $\tau_{1 s t}$ were in good agreement with their corresponding experimental values. The origin of such a large $\mathrm{L}$ is due to the return current flowing in a large, braided conductor located about $15 \mathrm{~cm}$ away from the discharge tube: this large-area current loop dominates the circuit inductance. For the discharge results presented in this paper, that current loop is essentially untouched and so $\mathrm{L}$ is essentially constant. The contribution of $\mathrm{R}_{\text {plasma }}$ appears to be a substantial contribution to $\mathrm{R}_{\text {int }}$, as we shall see later.

Figure 1(b) shows the three diagnostics discussed in the previous Section. The discharge parameters are identical to those just above, but with the addition of a series resistors $\mathrm{R}_{\mathrm{ext}}=16 \Omega$. Shooting off-screen on this image from an oscilloscope is the negative voltage pulse applied to the cathode. The middle trace shows the current in this critically-damped RLC circuit $(\mathrm{d}=0.99)$. The bottom trace shows the broadband optical emission collected from the optical fiber. The weak pulses seen at early times in the voltage and emission traces are from a preionization stage, which we will address in a later publication. For this configuration, such preionization did not affect the timing, magnitude, or jitter of the main waveforms.

From these traces, it appears that the rise in the current and in the emission occur within 50ns of the onset of the pulse applied to the cathode (after correcting for the different cable lengths of the three diagnostics). One would expect that the maximum rate of plasma heating occurs at the peak of the current pulse, yet the optical emission is dropping at that time. This is probably due to the depletion of neutral Ar. Singly-ionized Ar has many fewer transitions in the optical. This is borne out by looking at the emission as the heating pulse turns off. The afterglow is much brighter as the plasma recombines through excited neutral-Ar states.

\section{DENSITY MEASUREMENTS}

The plasma density was obtained by measuring the Stark-broadening of neutral line emission from the $\mathrm{H}_{2}$ dopant. In particular, the density-dependent broadening of the hydrogen Balmer $\alpha$ line at $6563 \AA$ was used (hereafter 
referred to a the H- $\alpha$ line). The three thin black curves in Fig. 2 show how the line widths (at FWHM) $\Delta \lambda$ of the Lorentzian-shaped $\mathrm{H}-\alpha$ line profiles vary with electron density.[6] The three curves represent three electron temperatures; $3.6 \mathrm{eV}, 1.8 \mathrm{eV}$, and $0.5 \mathrm{eV}$ moving from top to bottom. The line profiles were measured on a spectrometer with a spectral resolution of about $0.9 \AA$ per CCD pixel. The CCD was the model PI-MAX 3 from Princeton Instruments. This camera has an image intensifier that can be gated down to 10's of ns, although a $200 \mathrm{~ns}$ gate was used for these experiments. The center of the discharge tube was imaged onto the slit of the imaging spectrograph with an $\mathrm{f} / 5$ collection lens and with a magnification of about 1:1. Although the slit direction corresponds to the radial direction of the tube, the field of view was limited and no obvious radial variation in the line profiles were observed.

Figure 2 also shows three experimentally measured densities. Each measurement corresponds to the density at the peak of the discharge current. The lowest density case, Case 1, shown in the magenta dot-dashed line corresponds to the critically-damped discharge of Fig. 1(b) with its $0.03 \mu \mathrm{F}$ capacitor. A charging voltage of $20 \mathrm{kV}$ producing $\mathrm{J}_{\max }=1.2 \mathrm{kA} / \mathrm{cm}^{2}$ and gave a density of about $1 \times 10^{16} \mathrm{~cm}^{-3}$ in $4 \mathrm{~T}$ of Ar. Increasing the capacitance by a factor of about 15 to $\mathrm{C}=0.44 \mu \mathrm{F}$ produced a nearly fully ionized plasma (dashed red line, Case 2) at a charging voltage of $9 \mathrm{kV}$. Here, $\mathrm{J}_{\max }=3.1 \mathrm{kA} / \mathrm{cm}^{2}$, achieved by removing the $16 \Omega$ external resistor. With such a large increase in $\mathrm{C}, \tau_{1 \mathrm{st}}$ increased to $2.4 \mu \mathrm{s}$ FWHM; the current does not scale linearly with $\mathrm{C}$ due to the increase in the L-C time constant. The calculated $\mathrm{R}_{\text {int }}$ for this case is just $1.9 \Omega$, down from $3.2 \Omega$ in the lower density (and supposedly colder) plasma of Case 1. The solid blue line, Case 3, corresponds to a discharge in a 1" OD tube but with the same circuit components as in Case 2 . The charging voltage $\mathrm{V}_{0}$ needed to obtain $\mathrm{J}_{\max }=1.1 \mathrm{kA} / \mathrm{cm}^{2}$ was $15 \mathrm{kV}$. From the waveform (not shown), it appears that by increasing the tube diameter, $\mathrm{R}_{\text {int }}$ dropped to $1.2 \Omega$. A drop is expected since the area of the plasma is larger. Preliminary data suggests that the Ar reaches near full ionization in this 1 " tube at $J_{\max }$ $=2 \mathrm{kA} / \mathrm{cm}^{2}$ using a charging voltage of $28 \mathrm{kV}$. However, insufficient dielectric insulation near the spark gap limited the number of shots at such a high voltage.

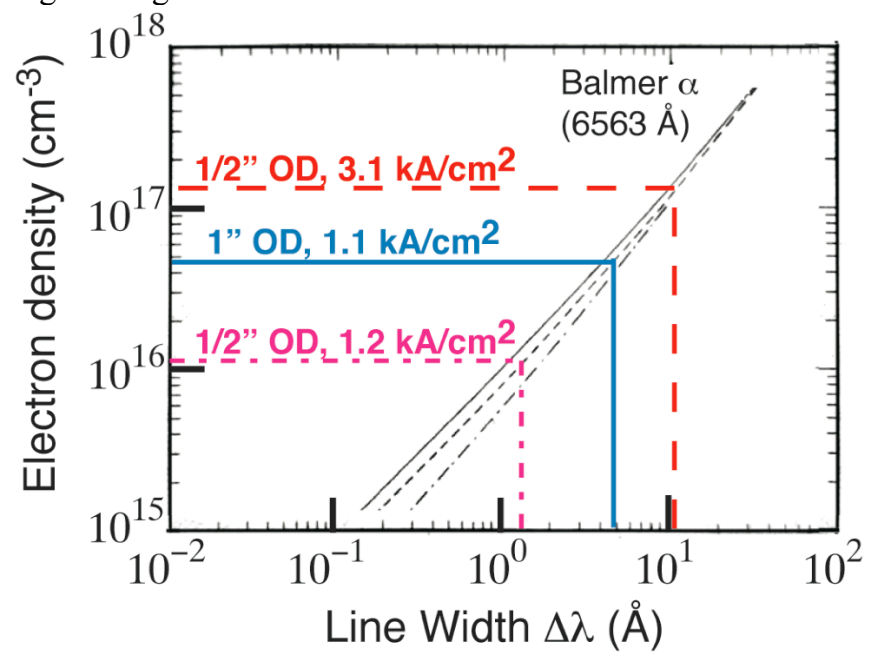

FIGURE 2. The theoretical electron density vs. the width of the Lorentzian-shaped hydrogen Balmer- $\alpha$ line $(H-\alpha$ line) at $6563 \AA$, taken from Ref. [6] (the three thin black curves). From top to bottom, these three curves correspond to plasma temperatures of about $3.6 \mathrm{eV}, 1.8 \mathrm{eV}$, and $0.5 \mathrm{eV}$, respectively. The three vertical lines correspond to the measured $\mathrm{H}-\alpha$ line-widths for: Case 1 (dot-dashed magenta line) used the same discharge parameters as in Fig. 1(b); i.e., a 1/2" tube, $\mathrm{C}=0.03 \mu \mathrm{F}, \mathrm{R}_{\mathrm{ext}}=16 \Omega$, and $\mathrm{V}_{0}=$ $20 \mathrm{kV}$ with a resultant $\mathrm{J}_{\max }=1.2 \mathrm{kA} / \mathrm{cm}^{2}$; Case 2 (dashed red line) with parameters of a $1 / 2$ " tube, $\mathrm{C}=0.44 \mu \mathrm{F}, \mathrm{R}_{\mathrm{ext}}=0 \Omega$, and $\mathrm{V}_{0}=$ $9 \mathrm{kV}$ with a resultant $\mathrm{J}_{\max }=3.1 \mathrm{kA} / \mathrm{cm}^{2}$; Case 3 (solid blue line) with parameters of a 1 " tube, $\mathrm{C}=0.44 \mu \mathrm{F}, \mathrm{R}_{\mathrm{ext}}=0 \Omega$, and $\mathrm{V}_{0}=$

$15 \mathrm{kV}$ with a resultant $\mathrm{J}_{\max }=1.1 \mathrm{kA} / \mathrm{cm}^{2}$. The approximate electron densities (horizontal lines) are taken from the central temperature (the actual temperature is unknown) giving densities of about 1,12 , and 5 in units of $10^{16} \mathrm{~cm}^{-3}$ for Cases 1,2 , and 3 , respectively.

Finally, a scan of the density vs. time in the 1" OD tube (Case 3) is shown in Fig. 3. A bad setting of the software delays is responsible for the zero of the data to be off by about twice the gate width of 200ns. However, the trending of the data is clear. The red diamonds show that $\Delta \lambda$, and thus the density, rises over about $2 / 3$ of the current (heating) pulse shown as the solid blue trace. Surprisingly, when the current crosses zero, there is little change in the density suggesting that losses to the wall are small and that recombination is limited by even a modest heating 
current. The dip in the amplitude of the Lorentzian fits to the $\mathrm{H}-\alpha$ line profiles (gray triangles) near the peak of the current may be evidence of loss of $\mathrm{H}$ atoms due to ionization. This amplitude climbs up again, as does the brightness of the Ar emission, as the plasma supposedly cools off somewhat near the first zero-crossing of the current. The data of Fig. 3 was taken out to $18 \mu \mathrm{s}$ and it is clear that the there is additional heating for the next two oscillations of the current pulse since the density goes up another $10-20 \%$ at these later times (not shown). However, it is not desirable to have the discharge circuit ring so this is primarily an academic point.

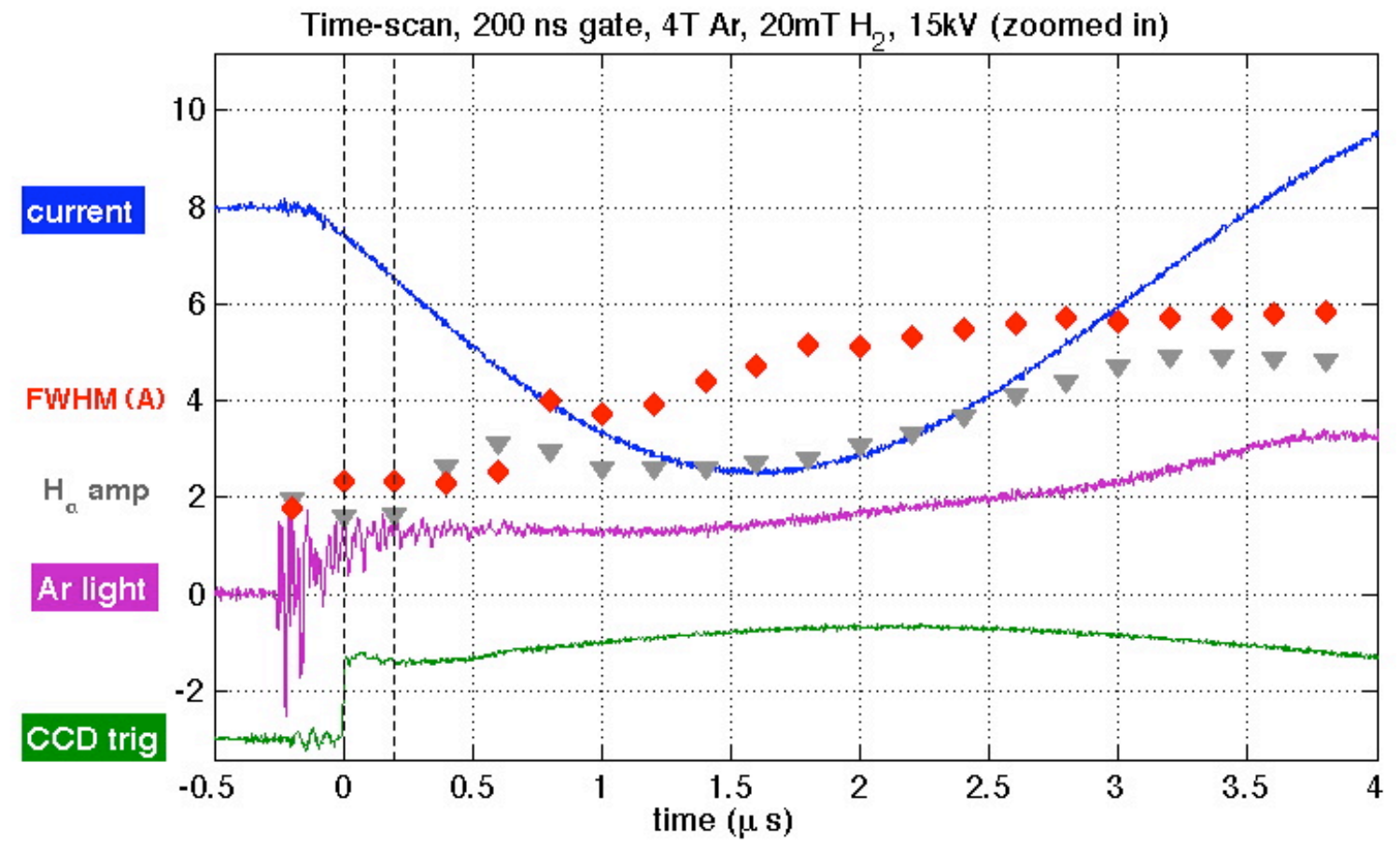

FIGURE 3. Three oscilloscope traces (see the labels along the vertical axis) showing the discharge current, the Ar optical emission, and a read-back of the CCD trigger, all for the Case 3, which was discussed with respect to Fig. 2 and detailed in the text. Also shown are the measured line widths $\Delta \lambda$ of the $H-\alpha$ line obtained from excellent Lorentzian curve-fits to the measured line profiles and averaged over 10 shots (red diamonds; labeled "FWHM ( $\AA$ )") as well as the amplitude of the same Lorentzian fits (grey triangles; labeled " $\mathrm{H}_{\alpha}$ amp"). The line emission was gated at 200ns and stepped in time by 200ns. Due to an unresolved error in the settings of the software delays of the CCD gate, the zero-timing is not known. However, it appears to be about 400ns earlier than indicated. The vertical scale is $1 \AA$ per unit for $\Delta \lambda$ and about $1.5 \mathrm{kA}$ per unit for the current. All other scales are in arbitrary units.

As a quick test of the axial uniformity of the plasma, images of the plasma were recorded from a distance of about $4 \mathrm{~m}$. Even at this distance, the $1.2 \mathrm{~m}$-long plasma was too long to avoid vignetting in the camera optics. Therefore, three images were recorded: (1) viewing the anode region; (2) viewing the central region; and (3) viewing the cathode region. Although the image of the central region showed the plasma brightness falling off toward either end, this was clearly due to vignetting since the images of either end showed that the fall off occurs only within roughly $5 \mathrm{~cm}$ from the cathode and anode electrodes. Recalling that these electrodes were $1 / 4$ " in diameter, it apparently takes about $5 \mathrm{~cm}$ to "fill" the 1" OD discharge tube with plasma; an observation that agrees with one's eye.

\section{CONCLUSIONS AND FUTURE WORK}

In conclusion, for the $1.2 \mathrm{~m}$-long discharge tubes discussed in this paper, it is possible to fully ionize $4 \mathrm{~T}$ of $\mathrm{Ar}$ in a $1 / 2$ " tube with a peak current density of about $3.1 \mathrm{kA} / \mathrm{cm}^{2}$ in a $3.4 \mu \mathrm{s}$ FWHM current pulse. Switching to a 1 " tube, we obtained about $40 \%$ ionization of $4 \mathrm{~T}$ of $\mathrm{Ar}$ at $1.1 \mathrm{kA} / \mathrm{cm}^{2}$ with a $2.3 \mu \mathrm{s}$ FWHM current pulse. Preliminary data suggest that by doubling this current, near full ionization can be achieved. It is thought, however, that full ionization is not absolutely necessary for the intended purpose of this plasma source.

The discharge circuit runs at $1 \mathrm{~Hz}$ with acceptable jitter ( $\pm 100 \mathrm{~ns}$ peak-to-peak) and it is anticipated that the total coulomb transfer of the spark gap may limit the number of shots. It is important, therefore, to minimize the duration 
of the current pulse. This can be easily done by re-routing the path taken by the return current. Vendor specifications suggest that this should not be an issue. For example, at $0.01 \mathrm{C} /$ shot for Case 3, a $100 \mathrm{kC}$-rated spark gap should last for more than one million shots. Replacing the spark gap with a grounded-grid thyratron will both reduce the jitter, increase the lifetime, and potentially allow for $>1 \mathrm{~Hz}$ operation.

Future work will be towards fashioning the discharge tube and electrodes to be compatible with a beam-driven wakefield accelerator experiment. Clearly, the electrodes must be hollow and of sufficient diameter as to allow for passage of the beam with or without the firing of the plasma. We are ready to test two $1.5 \mathrm{~m}$-long discharge tubes having inner diameters of 1 " and 1.5" with hollow electrodes of diameters 3/4" and $11 / 4$ ", respectively. The radial and longitudinal density profiles will be mapped out. Finally, in the application environment, there will almost certainly be a grounded conductor, effectively another anode, located closer to the cathode than the anode proper located $1.5 \mathrm{~m}$ away. At our operating pressures, the Paschen curve suggests that the current will take the shorter path. However, by adding intermediate electrodes and/or preionization, it may be possible to induce or force the discharge to take the "longer path". This will potentially be a major effort of the future work.

\section{ACKNOWLEDGMENTS}

This work was supported by DOE grant DE-FG02-92ER40727, NSF grant PHY-0936266, and FCT - Portugal, under grant PDCT/FIS/71709/2006.

\section{REFERENCES}

1. P. Muggli et al., Nature, 411, p. 43, May (2001).

2. I. Blumenfeld et al., Nature 445, 741-744 (2007).

3. M. J. Hogan et al., New Journal of Physics 12, 1-19 (2010).

4. N. Vafaei-Najafabadi et al., "Meter Scale Plasma Source for Plasma Wakefield Experiments", in these proceedings.

5. D. Bruce Montgomery, Solenoid Magnet Design, New York, Wiley-Interscience, John Wiley \& Sons, 1969, pp. $190-198$.

6. B. Bekefi, C. Deutsch, and B. Yaakobi, "Diagnostics of Laser Plasmas" in Principles of Laser Plasmas, edited by G. Bekefi, New York, Wiley-Interscience, John Wiley \& Sons, 1976, pp. 549-641. 